\title{
The OCHIN Community Information Network: Bringing Together Community Health Centers, Information Technology, and Data to Support a Patient-Centered Medical Village
}

\author{
Jennifer E. DeVoe, MD, DPhil, and Abigail Sears, MHA, MBA
}

Creating integrated, comprehensive care practices requires access to data and informatics expertise. Information technology (IT) resources are not readily available to individual practices. One model of shared IT resources and learning is a "patient-centered medical village." We describe the OCHIN Community Health Information Network as an example of this model; community practices have come together collectively to form an organization that leverages shared IT expertise, resources, and data, providing members with the means to fully capitalize on new technologies that support improved care. This collaborative facilitates the identification of "problem sheds" through surveillance of network-wide data, enables shared learning regarding best practices, and provides a "community laboratory" for practice-based research. As an example of a community of solution, OCHIN uses health IT and datasharing innovations to enhance partnerships between public health leaders, clinicians in community health centers, informatics experts, and policy makers. OCHIN community partners benefit from the shared IT resource (eg, a linked electronic health record, centralized data warehouse, informatics, and improvement expertise). This patient-centered medical village provides (1) the collective mechanism to build community-tailored IT solutions, (2) "neighbors" to share data and improvement strategies, and (3) infrastructure to support innovations based on electronic health records across communities, using experimental approaches. (J Am Board Fam Med 2013;26: 271-278.)

Keywords: Community Health Care, Electronic Health Records, Health Information Technology, Practice-based Research Network, Patient-Centered Medical Home

The 1967 Folsom Report, entitled "Health is a Community Affair," called for the provision of high-quality comprehensive personal health ser-

This article was externally peer reviewed.

Submitted 31 August 2012; revised 23 January 2013; accepted 4 February 2013.

From OCHIN, Inc., Portland, OR (JED, AS); and the Department of Family Medicine, Oregon Health and Science University, Portland (JED).

Funding: The development of research infrastructure at OCHIN was supported by grant no. 1RC4LM010852 from the National Institutes of Health (NIH) National Library of Medicine and grant no. UB2HA20235 from the Health Resources and Services Administration (HRSA). The data analyses that informed examples of measurement in this article were supported by grant no. R01 HL107647 from the NIH National Heart, Lung and Blood Institute.

Disclaimer: The funding organizations had no involvement in the design, preparation, review, or approval of this article.

Conflict of interest: JED is the executive director of OCHIN's practice-based research network. AS is the chief executive officer of OCHIN, Inc. vices to all people in each community. ${ }^{1}$ More than 50 years later, patient-centered medical homes (PCMHs) are being created as a way to achieve this aim. The joint set of principles defining the PCMH laid out a framework to guide the coordination and integration of care as well as continual measurement of quality and improvement outcomes. ${ }^{2}$ To be successful in efforts to create an integrated, comprehensive care practice, $\mathrm{PCMHs}$ need to harness data. This requires information technology (IT), data resources, and sophisticated informatics-expertise not readily available in most primary care practices, especially community health centers (CHCs) and other safety net organizations. There is concern that requirements for the "meaningful

Corresponding author: Jennifer E. DeVoe, MD, DPhil, OCHIN, Inc., 1881 SW Naito Parkway, Portland, OR 97201 (E-mail: devoej@ohsu.edu). 
use" of electronic health records (EHRs) could widen disparities and leave safety net populations behind. $^{3-5}$

One model to ensure IT support, data, and shared learning among community providers aiming to become PCMHs and to reach EHR meaningful use goals is that of an IT collaborative that serves as the hub for a "patient-centered medical village." We describe the OCHIN Community Health Information Network as an example of this model: community health centers, advocacy organizations, and patients from multiple communities have come together collectively to form an organization that leverages shared IT expertise, resources, and data, providing them with the means to capitalize fully on new technologies that support improved care. OCHIN embodies a community of solution (COS) that supports the provision of highquality comprehensive personal health services to individual patients while also using its collective health IT and data resources to share knowledge and information across multiple PCMHs and public health organizations. This dual function highlights two of the grand challenges (nos. 2 and 13) outlined by the Folsom Group in 2012. ${ }^{6}$ The OCHIN patient-centered medical village demonstrates a model of partnerships between patients, community advocacy organizations, public health leaders, CHC clinicians, informatics experts, and policy makers. These partners worked together to create shared IT infrastructure (eg, a linked EHR and centralized data warehouse and analytics coupled with informatics and improvement expertise). OCHIN's centralized IT resource facilitates the identification of "problem sheds" through surveillance of network-wide data, enables shared learning regarding best practices, and provides a "community laboratory" for practice-based research. Using experimental approaches, this COS model of a patient-centered medical village provides (1) the collective mechanism to build community-tailored IT solutions, (2) "neighbors" to share data and improvement strategies, and (3) infrastructure to support EHR-based quality improvement innovations across communities. In this model, the whole is greater than the sum of the parts.

\section{Phase 1: Creating OCHIN and Building the IT Foundation}

In 2001, CHCs governed by patient boards, community advocates, and other safety net organiza- tions from multiple communities formed a member-based, nonprofit collaborative, originally called the Oregon Community Health Information Network (renamed "OCHIN, Inc." as members from other states joined), to facilitate adopting health IT and a learning environment to improve care quality for vulnerable populations. This community collaborative enabled shared purchasing by multiple organizations, making it possible for the network to purchase an EHR that would have been unaffordable for individual members on their own. OCHIN is a nonprofit 501(c)(3) governed by a community board of directors, the majority of whom are community members of the collaborative. OCHIN provides and maintains a comprehensive electronic health information infrastructure for patient data through an Organized Health Care Arrangement, which is recognized in the Health Insurance Portability and Accountability Act (HIPPA) privacy rules and allows $\geq 2$ covered entities participating in joint activities to share protected health information about their patients to manage and benefit their joint operations. This arrangement gives patients receiving care from any OCHIN clinic a single medical record number, allowing clinical and utilization data to "follow the patient" to any OCHIN clinic. Member clinics share OCHIN's fully integrated EHR, which is built on EpicCare software (Epic Systems Corp, Verona, WI) and includes a practice management data system (claims, billing, scheduling) and an EpicCare electronic medical record.

Among the first major challenges in building OCHIN was choosing an EHR vendor and negotiating the initial EHR purchase. The centralized, integrated, technical model of EpicCare was a good fit for the OCHIN business model, which is centrally hosted. It was also important to find a vendor that was willing to be a partner amenable to making system modifications and enhancements for the organization. In addition, OCHIN wanted to maintain full access to all the network's EHR data, preferably through a library of reports accompanied by an easy-to-use query tool for nontechnical users. EpicCare did not have this function, so OCHIN helped members to fill this gap by identifying supplemental products.

The OCHIN collaborative now includes nearly 70 members with $>300$ clinic sites, providing a broad spectrum of primary health care services (eg, preventive care, public health, maternal and child health, behavioral/mental health, school-based services, den- 
Figure 1. Map showing the location of OCHIN member clinics (dots).

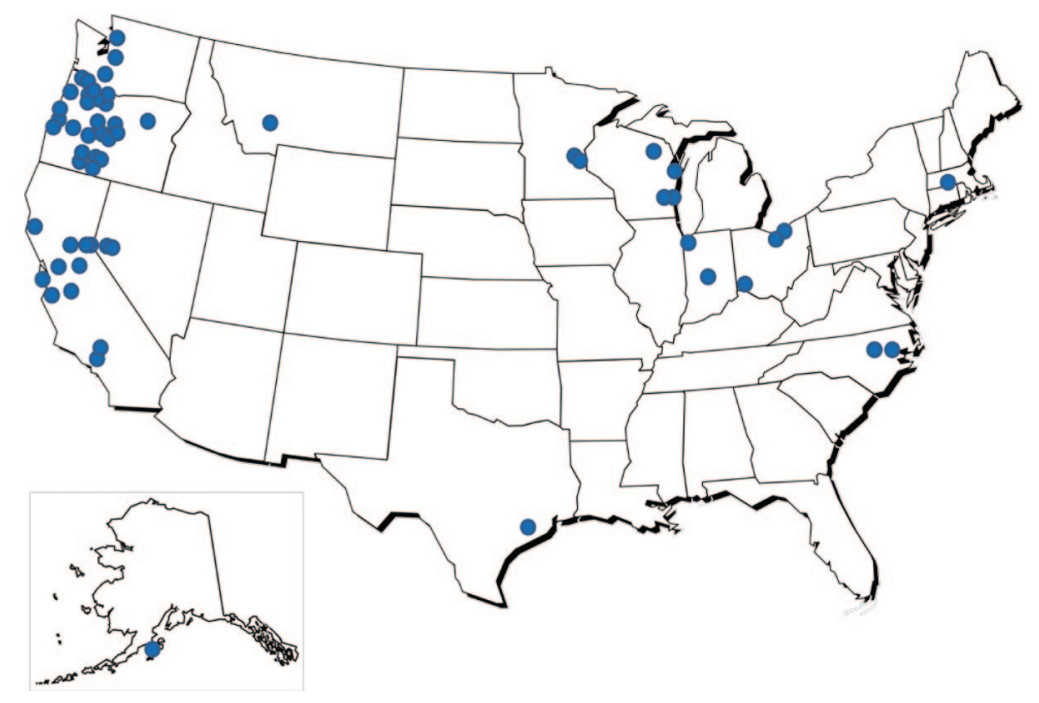

tal services, and care based in corrections facilities); see Figure 1 for a map of locations of current OCHIN members. Most OCHIN members are federally qualified health centers, rural health centers, and school-based health centers. OCHIN member clinics care for more than 1 million patients annually with more than 3 million visits. Some of the vulnerable populations served by these clinics include uninsured and underinsured patients, racial and ethnic minorities, migrant and seasonal farm workers, and patients living in poverty.

Because the EHR is centrally hosted at OCHIN and linked across all member clinics, each patient has a single medical record that is accessible to providers across the collaborative via an enterprisewide master patient index. There are many advantages to the shared EHR, including the seamless exchange of information between all OCHIN member clinics, which improves communication, integration, and coordination of care for complex patients and migrant populations. Data can also be centrally collected for quality improvement, public health surveillance (eg, tracking community influenza outbreaks), population health initiatives, research, and reporting purposes. The EHR data are standardized across sites and checked regularly for accuracy as part of OCHIN's member support.

While the diversity of the collaborative is an asset, it also posed some challenges such as the need to harmonize different clinical, billing, and reporting structures across the network and to achieve information-sharing agreements that complied with HIPPA and other privacy regulations. There are economies of scale to be gained by pooling and sharing resources, yet each member organization has its own needs and wants. OCHIN has learned that the tenets of the Pareto principle hold true when bringing new members into the collaborative and serving their needs: in most cases, approximately $80 \%$ of a new member's needs can be met by the collective resources; however, individualized and customized products are required to meet the additional 20\%. Managing these collective and individual needs is required both during "go-live" EHR installations and on an ongoing basis through continued support, training, and enhanced IT tools. This requires giving constant attention to needs, prioritizing projects, and using a skilled workforce. OCHIN has learned to offer products and services that are flexible to changing clinic needs and adaptable to the changing health care policy environment. This requires sophisticated change management approaches and peer interventions to help members achieve the best outcomes. OCHIN has developed internship and apprenticeship programs necessary to train and maintain personnel with the highly technical skills required to support this patient-centered medical village. It takes several years to build the interdisciplinary skills necessary to coach and mentor practices in the adoption of new IT tools and associated workflow changes. These important partnerships and training opportunities have strengthened the COS. 
During this first phase of OCHIN's development, we built community IT infrastructure, data resources, and a skilled IT workforce that provided a solid foundation for moving together collaboratively to enhance tools, data extraction, and shared learning across the patient-centered medical village.

\section{Phase 2: Enhanced Data Systems, Governance, Shared Learning, and Practice-based Research Enhanced Data Systems}

The centralized OCHIN infrastructure enabled the customization of a shared EHR platform and the development of EHR-enhancing tools. OCHIN optimizations to meet the needs of unique populations within the larger community included tailored systems for revenue management and billing (eg, charity programs, Medicaid), federal and state reporting (eg, Uniform Data System measures collected by the Health Resources and Services Administration), interoperability development, and integrated services (eg, OCHIN's Behavioral Health navigator to support the integration of primary care and mental health services). The OCHIN collaborative also facilitated interfaces to supplement the EHR with external information (eg, laboratory tests, images) and secure data interchange with federal and state agencies (eg, federal Social Security Administration, state Medicaid, state immunization registry). These efforts were focused largely on building interfaces that best supported integrated and coordinated care by facilitating the movement of data from primary care, mental health, and public health providers on the behalf of the patient. These external connections were vital for PCMHs serving populations that also were receiving services at other sites (eg, patients receiving immunizations at the public health department).

As OCHIN evolved, we recognized the need to maintain a network-wide electronic data warehouse that captured, stored, and maintained all data from the EHR. To optimize the use of this data in providing high-quality, integrated, comprehensive personal health services to patients, there was a need for supplemental software to aggregate data elements from the EHR warehouse and present the results in a user-friendly format. OCHIN helped to develop an open-source reporting tool that aggregates data from multiple locations in the EHR and presents the results in a common format, enabling providers to manage quality improvement. This data aggregator is implemented in standard SQL and contains a library of metrics that can be expanded by the user. A harvesting engine gathers necessary fields from electronic data sources including practice management, billing, medical records, laboratory, claims, disease management systems, public health reporting, and pharmacy data. It includes custom extractors to retrieve data from these systems, but data can also be entered directly. The harvesting engine includes tools to clean the data and assist with merging records. A calculation engine uses tables from the harvesting engine to produce metrics and build unique tables for creating registries and identifying patients in need of services. With the shared resources at OCHIN, it was possible to tailor this software for use in community settings, enhance its data warehouse and calculating functions, add a business intelligence interface, and train clinicians and staff across the collaborative to use it for clinical decision support, reporting, and other panel management activities.

Having the ability to extract and aggregate EHR data across a network of PCMHs also supports public health surveillance and population health planning. It enables the identification of problem sheds, whether they be geographic locations, racial/ ethnic groups, certain chronic diseases, or other populations in need of more intense assistance and intervention. This information can be shared with multiple partners in the collaborative, including public health agencies and other state and federal offices responsible for monitoring the health of special populations and targeting resources to where they are needed most.

\section{Governance and Advisory Groups}

OCHIN has a board of directors from communities across the country that makes major business decisions. However, a unique feature of the collaborative is the process through which members have input on the shared EHR, the further development of clinical decision support tools, and the ways that data are used. OCHIN hosts regular meetings of the Clinical Operations Group (COG), which comprises clinical leaders from all member organizations. Members of this group, some of whom have been working together for $>8$ years, review proposed changes to the shared EHR and guide quality improvement activities and population health initiatives across the collaborative. Executive leaders from the membership meet together twice yearly as the Executive Strategic Over- 
sight Council (ESOC) to provide strategic direction regarding issues that affect clinic operations. The COG and ESOC are chaired by members and traditionally have made decisions by consensus. As the collaborative has expanded to its current size, making decisions by consensus has become more challenging, and there is movement toward the election of a representative advisory council that would be smaller in size, meet more frequently, and have the ability to assist staff with efficient and nimble prioritization and optimization. The organization is moving cautiously in this direction so some of the smaller member clinics do not become marginalized.

\section{Shared Learning About Best Practices}

Not only do members share a common EHR hosted by OCHIN, these practices are all transforming into PCMHs and striving to meet EHR "meaningful use" goals. Within the OCHIN collaborative, individual clinics already are engaged in cutting-edge innovations to meet local, state, and national goals. The regular COG and ESOC meetings have enabled members to come together and share best practices and lessons learned regarding workflow changes needed to realize PCMH goals. The collaborative and its member groups also provide a structured environment with peer mentors and a shared EHR to enable practices to come together, share their individual innovations, and spread them across the network. In other words, this patient-centered medical village provides the platform to support, facilitate, scale up, and spread what many clinics are already doing. It also creates the energy and buoyancy to lift members to the next level of improvement and practice transformation. Furthermore, by using health IT and datasharing across the network, relevant knowledge can flow more widely and efficiently to support action planning for community health services, as recommended in the original Folsom Report in 1967 and highlighted as grand challenge no. 13 by the Folsom Group in 2012.1,6

\section{Practice-based Research}

In this phase of OCHIN's development, there was recognition from key leaders and stakeholders that this organization was uniquely positioned as a "community laboratory" for practice-based research that could inform practice and policy change. With funding from the American Recovery and Reinvestment Act, OCHIN has developed a robust practice-based research infrastructure, partnering with academic researchers interested in studying innovations across the network. The OCHIN SafetyNetWest practicebased research network (PBRN) is registered with the US Agency for Healthcare Research and Quality. ${ }^{7,8}$ Clinicians and researchers affiliated with the PBRN meet monthly to review and provide feedback on proposed research projects that would take place in member clinic sites, use OCHIN data, require other network resources, or all three. The newly-formed OCHIN Patient Engagement Panel (PEP) serves the researchers and clinicians of the PBRN as a patient voice in research; the PEP works with the PBRN to provide the patient perspective on grant proposals, proposed research methods (especially patient recruitment), compensation, and dissemination.

The OCHIN research team is engaged in a variety of research projects, including the analyses of large datasets to study the impact of practice and policy changes, the development of natural language processing software, and the translation of clinical and system interventions from integrated care settings into safety net clinics. The OCHIN collaborative also provides a unique "community laboratory" for conducting dissemination and implementation research because it can support cluster randomized trials that test system and IT interventions, such as the introduction of new electronic clinical decision support tools or new quality improvement strategies.

\section{Measuring the Progress of the OCHIN Community of Solution}

There are a couple of ways in which the progress of this unique COS can be measured. First, the OCHIN collaborative is committed to helping members achieve stage 1 EHR meaningful use goals by building and installing IT systems, helping practices stabilize and optimize those systems, and training clinic staff to use these systems to their fullest capabilities. OCHIN plans to continue supporting members in their goals to reach EHR meaningful use stage 2 and 3 benchmarks as early as possible; these achievements can be measured. Similarly, we can measure the success of member practices in meeting PCMH standards, especially those most relevant to EHR and data use. Second, the OCHIN data repository allows for longitudinal measurement of numerous quality metrics as a means to follow trends over time and to scan across member clinics to identify those that are most in 
Figure 2. Variability in the quality of diabetes preventive care across the network. Data shown as percentages of patients receiving diabetes preventive services. FLU, influenza vaccination; HbA1c: glycohemoglobin screening; LDL, low-density lipoprotein cholesterol screening; Micro-Alb, urine microalbumin (nephropathy screen).

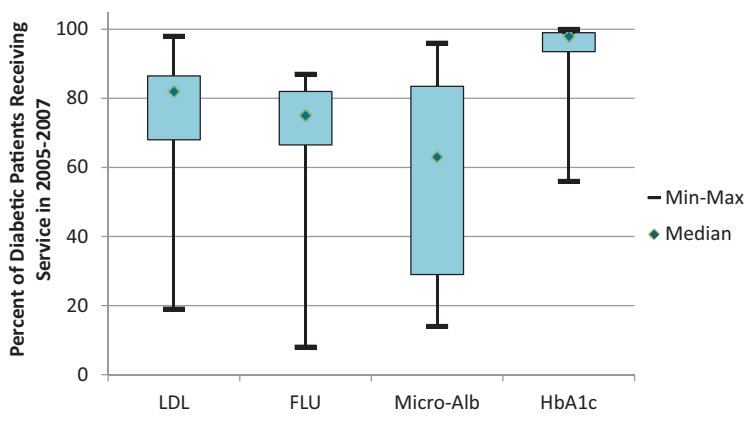

need of assistance (and others that can offer assistance). This approach to identifying problem sheds and measuring success enables OCHIN to use its data warehouse to conduct surveillance across the network. For example, in one analysis of networkwide data from 2005 to 2007, we found wide variation among diabetics receiving preventive care services across our network (Figure 2). ${ }^{9}$ This surveillance capability allows us to identify which sites are doing well, study their approach and processes, and then spread those lessons to sites that are struggling. It also enables us to better understand how the demographics of patient populations differ between sites and which characteristics are most predictive of variations in performance. This information is highly relevant in shaping policies that continue to incentivize clinics to care for these vulnerable populations rather than penalize clinics for doing so.

A measurement approach that targets meeting both individual and collective improvement goals is important to assess the impact of our patient-centered medical village on improving the health of vulnerable populations and eliminating health care disparities. It also pushes the paradigm shift one step further, from treating individual patients to managing a community health center patient panel to improving the care of populations across a network of CHCs. ${ }^{6}$

The OCHIN model of a centralized data warehouse coupled with a team of highly skilled informatics and analytics experts provides resources for member practices beyond what each could do in- dividually. It does not, however, require the individual entities to merge or be subsumed into a larger integrated system. This collaborative model between independent organizations allows each to maintain autonomy to innovate in ways that address the diverse communities and cultures they serve. Individual members also play a central role in the governance of the organization and common ownership in the health IT tools, processes, and relationships that bind members together with a mission to serve the underserved (Figure 3). If PCMHs are to be truly successful, clinics cannot go it alone; they must have shared IT and data resources coupled with meaningful opportunities to share best practices and get support from colleagues.

\section{Phase 3: Future Vision}

As the OCHIN collaborative continues to grow, we hope to add the following enhancements in the near future: (1) patient engagement in setting priorities and (2) a longitudinal fellowship training opportunity for member clinicians.

\section{Engaging Patients Through a Virtual Network}

As OCHIN continues building the infrastructure for a patient-centered medical village, the new IT tools supporting this collaborative will also enable more meaningful engagement of patients in efforts to improve quality of care. Modeled after the Public Insight Networks in the media industry, we plan to create the OCHIN Patient Engagement Network, designed as a virtual network of patients across the country who regularly will contribute their important perspectives to the collective "bank of insights" guiding the development of patientcentered improvements, measurement, evaluations. This will be accomplished by recruiting a national cohort of patients for remote engagement in research and priority-setting via the personal health record/patient portal in the OCHIN EHR. Patient participants in the OCHIN Patient Engagement Network will have the opportunity to participate in ongoing communication via a private blog and will be invited to complete brief (3 or 4 questions) electronic surveys on a regular basis to solicit their perspectives on topics and issues important to research and quality improvement. The virtual network will be designed and facilitated in close collaboration and partnership with our PBRN patient engagement panel. This vision aligns with the Folsom 
Figure 3. The OCHIN patient-centered medical village model enables a linked electronic health record, shared health information technology (IT) infrastructure, and collaborative learning across patient-centered medical homes (PCMHs) and other health care entities.

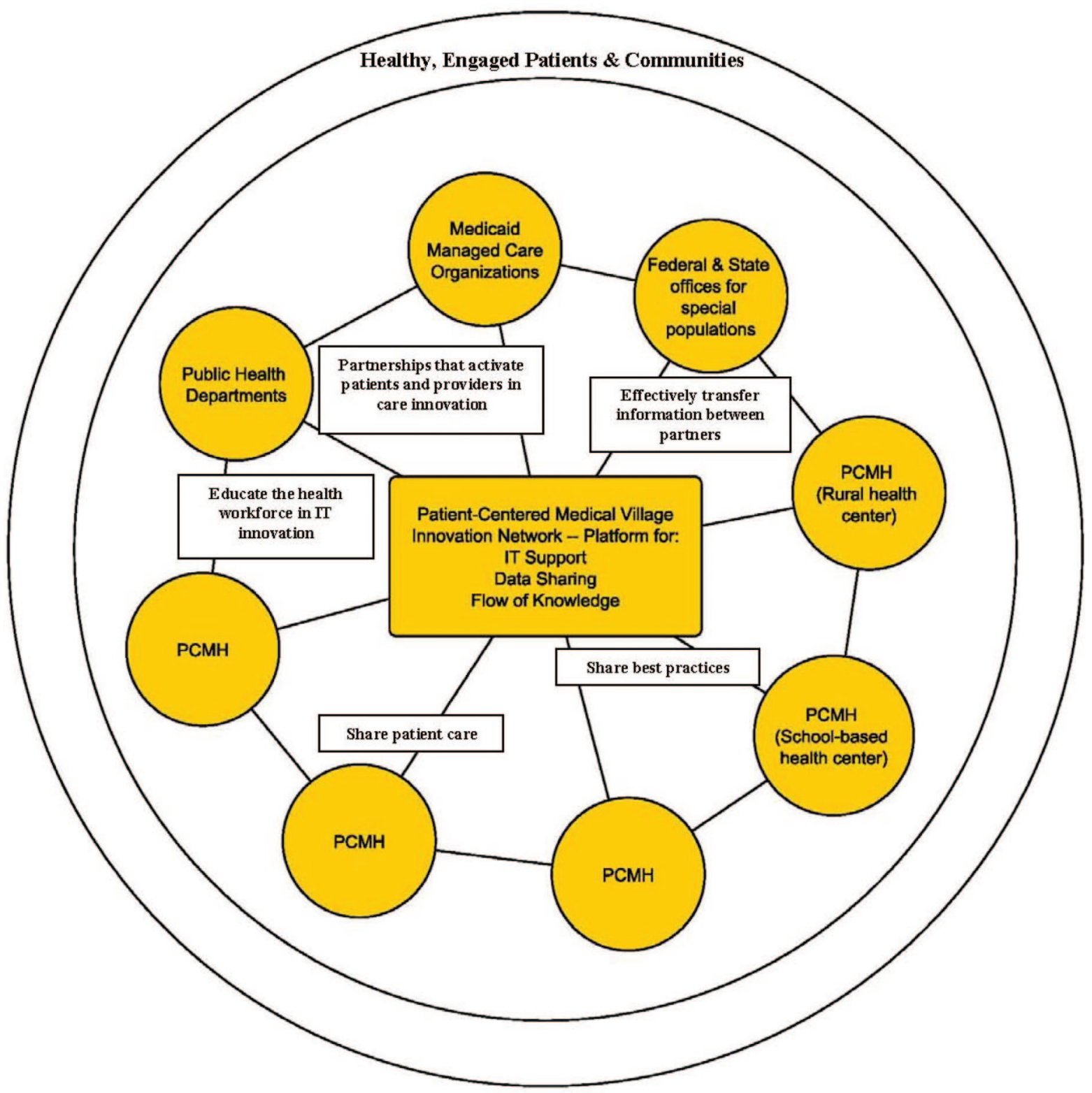

Report recommendation to organize and deliver community health services outside of traditional boundaries or political jurisdictions and addresses grand challenge no. 1 put forth by the Folsom Group by using IT resources to create a national platform for engagement and activation of patients. ${ }^{1,6}$

\section{Longitudinal Fellowship Training for Physicians and Community Health Teams}

A patient-centered medical village, such as the one we are building at OCHIN, needs "village cham- pions." Similar to "clinician champions," who are key to the success of practice-based research and quality improvement projects, we plan to expand on our internship programs to create specialized training for individuals willing to lead and facilitate collaborative sharing and the spread of ideas across the village. Rather than remove leaders from their communities to participate in a full-time fellowship, we envision creating longitudinal part-time opportunities for clinicians to stay grounded in their $\mathrm{CHCs}$ while simultaneously participating in a 
cohort of fellows to work in an apprenticeship-type arrangement. In this model, fellows would routinely travel to the "hub," helping to build the village while expanding their toolkit of IT and quality improvement skills for village leadership in the future. Many of these skills are what the Folsom Report described as necessary for the "ideal primary care physician" who is "the central point for integration and continuity of all medical and related services."1,6 This fellowship would include some of the traditional training in public health, improvement science, and informatics degree programs, but it would also capitalize on hands-on learning while sitting elbow-to-elbow with information architects, workflow engineers, systems scientists, research analysts, and improvement experts. Learners would bring new ideas and infuse enthusiasm into activities happening at the hub; thus, the flow of information, knowledge, and energy would be bidirectional. By contributing to the future health care workforce and enhancing the ability of a team of health professionals to use integrated community health services, this addresses the Folsom Group's grand challenge nos. 3 and $9 .^{6}$

\section{Conclusion}

The patient-centered medical village model creates an IT hub to support the development of integrated, comprehensive care practices and action planning for community health services. If organized well, a patient-centered medical village can meet many clinical and organizational goals while shifting the focus to improving population health collectively. Capitalizing on a linked EHR, shared IT infrastructure, and collaborative governance structure, the OCHIN patient-centered medical village was created to have meaningful impact on reducing health care disparities. This model is also designed to engage clinicians and patients in collaborative efforts to improve population health. As this model evolves and community members learn to harness the power of the collective data resources and learning collaborative, it is hoped that shared IT resources and data will be used more fully and effectively to support the provision of high-quality, comprehensive, personal health services, improved community health and action planning for community health services, and policy advocacy.

The authors gratefully acknowledge the contributions of Sonja Likumahuwa, Jon Puro, Jill Arkind, Rachel Gold, Vance Bauer, Christine Nelson, Steffani Bailey, and Jean O'Malley.

\section{References}

1. National Commission on Community Health Services. Health is a community affair. Report of the National Commission on Community Health Services (NCCHS). Cambridge: Harvard University Press; 1967.

2. Stenger R, DeVoe JE. Policy challenges in building the medical home: do we have a shared blueprint? J Am Board Fam Med 2010;23:384-392.

3. Office of the National Coordinator for Health Information Technology. Federal health information technology strategic plan 2011-2015. Available from http:// www.healthit.gov/policy-researchers-implementers/ health-it-strategic-planning. Accessed March 13, 2013.

4. Hing E, Burt CW. Are there patient disparities when electronic health records are adopted? J Health Care Poor Underserved 2009;20:473-88.

5. Sequist TD. Health information technology and disparities in quality of care. J Gen Intern Med 2011;26: 1084-5.

6. Folsom Group. Communities of solution: the Folsom Report revisited. Ann Fam Med 2012;10:250-60.

7. Devoe JE, Gold R, Spofford M, et al. Developing a network of community health centers with a common electronic health record: description of the Safety Net West Practice-based Research Network (SNWPBRN). J Am Board Fam Med 2011;24:597-604.

8. DeVoe JE, Likumahuwa S, Eiff MP, et al. Lessons learned and challenges ahead: report from the OCHIN Safety Net West practice-based research network (PBRN). J Am Board Fam Med. 2012;25: 560-4

9. DeVoe J, Gold R, O’Malley J, Bailey S, Puro J, McIntire P. Variability in care quality: do federally qualified health center patient demographics correlate with quality of diabetic care? Presented at the AcademyHealth Annual Research Meeting, June 24-26, 2012, Orlando, FL. 